\title{
Diagnostic roles of urinary kidney microvesicles in diabetic nephropathy
}

\author{
Fang-Hao Cai ${ }^{1,2}$, Wen-Yan $\mathrm{Wu}^{1,2}$, Xu-Jie Zhou ${ }^{1,2}$, Xiao-Juan Yu ${ }^{1,2}$, Ji-Cheng Lv $^{1}$, Su-Xia Wang ${ }^{1,2,3}$, \\ Gang Liu ${ }^{1,2}$, Li Yang ${ }^{1,2} \wedge$ \\ ${ }^{1}$ Renal Division, Peking University First Hospital, Peking University Institute of Nephrology, Key Laboratory of Renal Disease, Ministry of Health \\ of China, Key Laboratory of Chronic Kidney Disease Prevention and Treatment (Peking University), Ministry of Education, Beijing, China; ${ }^{2}$ Renal \\ Pathology Center, Peking University First Hospital, Beijing, China; ${ }^{3}$ Laboratory of Electron Microscopy, Ultrastructural Pathology Center, Peking \\ University First Hospital, Beijing, China \\ Contributions: (I) Conception and design: L Yang; (II) Administrative support: L Yang; (III) Provision of study materials or patients: FH Cai, WY \\ Wu; (IV) Collection and assembly of data: FH Cai; (V) Data analysis and interpretation: FH Cai, XJ Zhou, XJ Yu, JC Lv, SX Wang, G Liu; (VI) \\ Manuscript writing: All authors; (VII) Final approval of manuscript: All authors. \\ Correspondence to: Dr. Li Yang, MD. Renal Division, Peking University First Hospital, Peking University Institute of Nephrology, Key Laboratory of \\ Renal Disease, Ministry of Health of China, Key Laboratory of Chronic Kidney Disease Prevention and Treatment (Peking University), Ministry of \\ Education, No. 8, Xishiku Street, Xicheng District, Beijing 100034, China. Email: li.yang@bjmu.edu.cn.
}

Background: The pathology of diabetic nephropathy (DN) broadly involves the injury of glomeruli, tubulointerstitium and endothelium. Cells from these compartments can release increased numbers of microvesicles (MVs) into urine when stressed or damaged. Currently whether urinary MVs from these three parts can help diagnose $\mathrm{DN}$ and reflect pathological features remain unclear.

Methods: Forty-nine patients with histologically proven DN and 29 proteinuric controls with membranous nephropathy or minimal change disease were enrolled. Urinary podocyte, proximal tubular and endothelial cell-derived MVs were quantified by flow cytometry. Renal glomerular, tubulointerstitial and vascular lesions were semi-quantitatively scored and their relevance to urinary MVs were analyzed.

Results: DN patients had greater numbers of urinary MVs from podocytes, proximal tubular and endothelial cells compared with proteinuric controls. The combination of podocyte nephrin + MVs and diabetic retinopathy optimally diagnose DN with $89.7 \%$ specificity and $88.9 \%$ sensitivity. Moreover, positive correlations were observed between urinary levels of proximal tubular MVs and the severity of tubular injury and between urinary levels of endothelial MVs and the degree of vascular injury. Using urinary proximal tubular MVs as the indicators for tubular injury, the differences between DN patients and proteinuric controls diminished after matching the degree of renal vascular injury or when proteinuria $>8 \mathrm{~g} / 24 \mathrm{~h}$.

Conclusions: Urinary kidney-specific cell-derived MVs might serve as noninvasive biomarkers for the diagnosis of DN in diabetic proteinuric patients. Their elevated levels could reflect corresponding renal pathological lesions, helping physicians look into the heterogeneity of DN.

Keywords: Diabetic nephropathy (DN); microvesicle; renal pathology; diagnosis

Submitted Jan 09, 2020. Accepted for publication Aug 20, 2020.

doi: $10.21037 / \mathrm{atm}-20-441$

View this article at: http://dx.doi.org/10.21037/atm-20-441

$\wedge$ ORCID: 0000-0002-2528-5087. 


\section{Introduction}

Diabetic nephropathy (DN) constitutes a large proportion of patients with chronic kidney disease (CKD) and contributes greatly to end stage renal disease (ESRD) $(1,2)$. Diagnosis of DN depends on kidney biopsy (3), and the pathology involves all compartments of the kidney including progressive podocyte injury (4), extensive endothelial impairment (5) and prominent tubulointerstitial abnormalities (6). With the continuous growth of diabetes epidemic, an increasing number of cases of diabetic kidney disease are seen (2). However, the renal pathology and underlying pathogenesis vary considerably, which impedes prompt and appropriate treatment. The aspects related to DN diagnosis are complicated which makes the diagnosis rather difficult in some cases. According to published studies, among diabetic patients who had kidney diseases and underwent renal biopsy $34.5-72.7 \%$ presented non-DN kidney injuries (7-11), of which membranous nephropathy $(\mathrm{MN})$ was most frequently seen $(24.1-32.2 \%)$ followed by minimal change disease (MCD, 6.9-16.7\%) (9-11). The high prevalence of non-diabetic renal disease in different centers indicates a strong need for noninvasive biomarkers to discriminate between these $\mathrm{DN}$ and nonDN disease conditions. Second, DN per se is heterogenous and histologically manifested as various pathological presentations. Noninvasive biomarkers reflecting kidney pathology could provide insights into DN progression individually.

Renal cells release substantial heterogeneous extracellular vesicles carrying cell-specific proteins into urine. Microvesicles (MVs) are medium size extracellular vesicles $(0.1-1 \mu \mathrm{m})$ formed by directly outward blebbing. The release of MVs is markedly increased when cells are stressed or injured $(12,13)$. Recently, studies have focused on urinary podocyte MVs and observed the increased levels in various kidney diseases affecting podocytes, such as type 1 diabetes with normoalbuminuria (14), renovascular hypertension (15) and preeclampsia (16). In addition, urinary endothelial MVs were elevated in hypertensive patients and negatively correlated with cortical perfusion (17). However, whether urinary MVs could help with differential diagnosis of renal diseases has not been investigated. By a cross-sectional study of 78 Chinese patients with biopsy-proven DN or proteinuric controls with $\mathrm{MN}$ or MCD, our study aimed to check the diagnostic value of urinary kidney MVs for DN and investigate their pathological relevance. We present the following article in accordance with the STARD reporting checklist (available at http://dx.doi.org/10.21037/atm-20-441).

\section{Methods}

\section{Patients}

The research was in compliance of the Declaration of Helsinki (as revised in 2013), and approved by the Committee on Research Ethics of Peking University First Hospital (NO. 20171280). All participants gave written informed consent. Patients with native renal biopsy-proven DN from August 1, 2017, to August 31, 2018 in our department were consecutively enrolled (Figure 1). DN was diagnosed according to Tervaert's glomerular classification (3). The enrollment criteria included (I) consent to the study and (II) valid urine samples for $M V$ analysis. Patients with concurrent primary or secondary proliferative glomerulonephritis, urinary tract infection or malignancy were excluded. Altogether, 49 cases of DN met the study entry criteria. Twenty-nine age-, sex- and proteinuria-matched patients with $\mathrm{MN}$ or MCD during the same study period were enrolled as proteinuric controls. Fourteen age- and sex-matched healthy volunteers were included as healthy controls.

\section{Renal pathology evaluation}

The standard processing of kidney biopsy specimens included light microscopy and electron microscopy. Two pathologists made the diagnosis and semiquantitative scores separately without knowledge of patients' clinical information.

In glomerular lesions, classification of DN was based on the criteria of Tervaert et al. (3). Microaneurysms, mesangiolysis and Kimmelstiel-Wilson nodules were recorded as being present or not. Mesangial expansion was semi-quantitatively scored as follows: 0 for absent, 1 for $<25 \%, 2$ for $25-50 \%$ and 3 for $>50 \%$ of the observed mesangium areas. Glomerular sclerosis and ischemic sclerosis were expressed as percentages.

Evaluation of renal tubulointerstitial lesions included tubular injury, interstitial inflammation, tubular atrophy and interstitial fibrosis. Tubular injury was scored as the sum of the presence of following lesions with a maximum score of 6 points: hyaline droplet change, vacuolar change, loss of brush border, interstitial edema and epithelial cell sloughing $(\mathrm{x} 2)$. Interstitial inflammation, tubular atrophy and interstitial fibrosis were scored semi-quantitatively as follows: 0 for absent, 1 for $<25 \%, 2$ for $25-50 \%, 3$ for $50-75 \%$ and 4 for $>75 \%$ of total tubulointerstitial areas. 


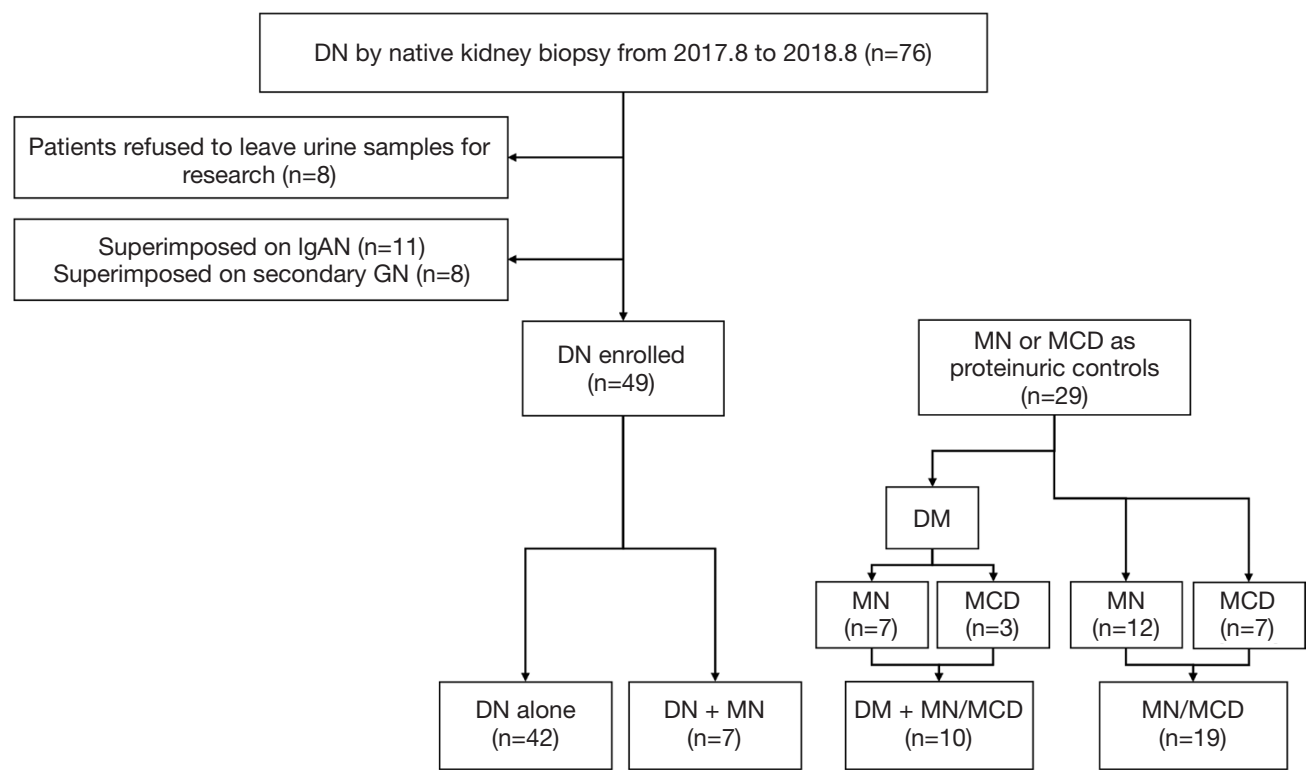

Figure 1 Flow chart of the patients enrolled in the study. DM, diabetes mellitus; DN, diabetic nephropathy; MN, membranous nephropathy; MCD, minimal change disease; IgAN, IgA nephropathy; GN, glomerulonephropathy.

The chronic tubulointerstitial injury score was the sum of the scores for tubular atrophy and interstitial fibrosis with a maximum score of 8 points.

The vascular score was defined as the sum of the scores for arteriolar hyalinosis and arteriosclerosis with a maximum score of 3 points. Arteriolar hyalinosis was designated as being present (score 1) or not. Arteriosclerosis was scored according to the most severely affected artery in the biopsy: 0 for absence of intimal thickening, 1 for intimal thickening with a thickness less than that of the media and 2 for intimal thickening with a thickness greater than that of the media.

\section{Urine sample collection}

First-morning urine samples were prospectively collected. For patients who underwent biopsy, urine samples were collected four days post-renal biopsy after excluding hemorrhage complications. The urine samples were centrifuged at 2,500 $\times \mathrm{g}$ for 10 minutes at $20{ }^{\circ} \mathrm{C}$ to remove cells and large debris. Proteases inhibitors were not added. The supernatants were stored at $-80{ }^{\circ} \mathrm{C}$ until assay.

\section{Flow cytometry analysis of urinary $M V s$}

MVs in cell-free urine were directly characterized and enumerated by flow cytometry according to the previously validated method $(16,18,19)$. Frozen urine samples were thawed in a $37^{\circ} \mathrm{C}$ water bath for 5 minutes. An initial $20 \mu \mathrm{L}$ volume was diluted in $80 \mu \mathrm{L}$ phosphate buffer saline (PBS) and incubated with $3 \mu \mathrm{L}$ annexin-V FITC for concentration check. The volume of urine in the subsequent experiment was then determined for yielding at least 1,000 annexin- $V$ positive urinary MVs and an event rate between 1,000 and 3,000 events/s.

Diluted urine samples of $100 \mu \mathrm{L}$ each were then stained for annexin- $\mathrm{V}$ and cell-specific markers. FITC-conjugated annexin-V (3:100; Becton, Dickinson and Company) was used as a MV marker. PE-conjugated anti-podocin antibody (1:200, Bioss) and PE-Cy7-conjugated anti-nephrin antibody (1:200, Bioss) were used to identify podocyte origin. PE-conjugated anti-megalin antibody (1:200, Bioss) and APC-conjugated anti-AQP1 (1:200, Bioss) antibody were used to identify proximal tubular cell origin. PEconjugated anti-CD144 antibody (3:100, BioLegend) and APC-conjugated anti-CD31 antibody (3:100, BioLegend) were used to identify endothelial cell origin. After incubation for 30 minutes in the dark, each sample was diluted with annexin- $\mathrm{V}$ binding buffer to a final volume of $1 \mathrm{~mL}$ prior to flow cytometry. MVs diluted in annexin- $\mathrm{V}$ binding buffer lacking calcium or containing PE, PE-Cy7 and APC-conjugated immunoglobulin G (IgG) control antibodies were considered negative controls. All reagents 
were filtered through a $0.22 \mu \mathrm{m}$ size filter (Millipore) twice.

A flow cytometer CytoFLEX (Beckman Coulter) was used to analyze urinary MVs. Size gates were established using standard Megamix beads of 0.16, 0.2, 0.24, 0.3, 0.5, 0.9 and $3 \mu \mathrm{m}$ (Diagnostica Stago). MVs were defined as events of $0.2-1 \mu \mathrm{m}$ in diameter and positive for annexin- $\mathrm{V}$ or cell-specific markers $(18,19)$. MVs were quantified by CytoFLEX automatically (20). The value of urinary MVs was expressed as the absolute number per $\mu \mathrm{L}$ of urine and per $\mu \mathrm{mol}$ of urinary creatinine.

\section{Transmission electron microscopy}

Pooled cell-free urine samples of $100 \mathrm{~mL}$ were centrifuged at 20,000 ×g (Rotor JA25.50, Beckman Coulter's Avanti J High-speed Centrifuge) for 20 minutes at $25{ }^{\circ} \mathrm{C}$ to get $\mathrm{MV}$-enriched pellet, where apoptotic bodies and exosomes could be coprecipitated. The resulting pellet was fixed overnight in $2.5 \%$ glutaraldehyde at room temperature, washed three times with $\mathrm{PBS}$, postfixed in $2 \% \mathrm{OsO}_{4}$ at $4{ }^{\circ} \mathrm{C}$ for $2 \mathrm{~h}$ and then washed twice with double distilled water. After dehydrated in graded acetone, the pellets were embedded in resin-812. Sections of $70 \mathrm{~nm}$ were prepared on copper grids and visualized on a JEM-100CX TEM microscope (JEOL).

\section{Statistical analysis}

SPSS 22.0 (IBM Corporation, New York) were used. Quantitative variables were expressed as the mean $\pm \mathrm{SD}$ or median with interquartile range (IQR, for data without a normal distribution). Differences between groups were analyzed using a $t$-test or Mann-Whitney $\mathrm{U}$ test with a Bonferroni posttest as appropriate. Categorical data were interpreted in the form of constituent ratios and were compared by the chi-square test. Correlations among the MV populations and pathological features were analyzed using Spearman correlation coefficient. For multivariate analysis, we used principal component analysis (PCA) to concentrate information, logistic regression and ordinal regression to uncover independent risk factors for DN and pathological features. Results were expressed as odds ratios (OR) with $95 \%$ confidence intervals (CI). The diagnostic performance was assessed by receiver operating characteristic (ROC) curves. Four DN patients without fundus photography for diabetic retinopathy were excluded for the related ROC curve analysis. Two-tailed $\mathrm{P}$ values $<0.05$ were considered statistically significant.

\section{Results}

\section{Demographic and clinical features of patients in different groups}

As shown in Figure 1, altogether 78 patients were enrolled including 42 with DN alone, 7 with DN superimposed on $\mathrm{MN}(\mathrm{DN}+\mathrm{MN})$ and 29 proteinuric controls with $\mathrm{MN}$ or MCD, among whom 10 were diabetic $(\mathrm{DM}+\mathrm{MN} /$ MCD) and the other 19 were nondiabetic (MN/MCD). The patients with $\mathrm{DN}$ alone were younger $(45.9 \pm 11.1$ vs. 58.6 $\pm 9.4, \mathrm{P}=0.016)$ and more likely to have diabetic retinopathy ( $75 \%$ vs. $30 \%, \mathrm{P}=0.021)$ compared to those in the DM+MN/MCD group, while no significant difference was detected in the length of diabetic history or blood glucose control between the two groups (Table 1). Among all groups, the patients with DN alone had the highest levels of systolic blood pressure, serum creatinine and serum albumin. There was no difference in the levels of proteinuria, urinary NAG, urinary $\alpha 1$-microglobulin or hematuria among the four groups, while urinary $\alpha 1$ microglobulin was found to be significantly higher in the total DN patients compared with the total proteinuric controls [23.8 (15.8, 69.5) vs. 47.3 (19.1, 111.0), $\mathrm{P}=0.049]$.

\section{Urinary MV levels in patients and bealthy subjects}

The morphology and size of structurally intact vesicles were checked by transmission electron microscopy (Figure 2A). Annexin- $\mathrm{V}^{+} \mathrm{MVs}$ were increased in all the patient groups (Figure 2B-D). The MV counts normalized to urine creatinine (MVs/U-Cr) had the ability to discern proteinuric patients rather than analyzed as a concentration $(\mathrm{MVs} / \mu \mathrm{L})$ (Figure $2 E$ ). Although annexin- $\mathrm{V}^{+} \mathrm{MVs}$ had the ability to identify proteinuric patients (area under the curve, AUC 0.775, 95\% CI: $0.626-0.923, \mathrm{P}=0.001$, specificity $78.6 \%$, sensitivity $80.8 \%$ ), it was not sufficient to discriminate the patients with various etiologies, i.e., between $\mathrm{DN}$ patients and proteinuric controls (Figure 2F).

\section{Urinary kidney MVs discriminate $\mathrm{DN}$ from proteinuric controls}

PCA analysis demonstrated that the comprehensive urinary MV feature divided patients into two groups, having $\mathrm{DN}$ and not (Figure 3A,B). And there was no significant difference in urinary levels of podocyte, proximal tubular and endothelial cell-derived MVs between the DN alone group and the $\mathrm{DN}+\mathrm{MN}$ group or between $\mathrm{MN} / \mathrm{MCD}$ 
Table 1 Demographic and clinical characteristics of patients in different groups

\begin{tabular}{|c|c|c|c|c|c|}
\hline & \multicolumn{2}{|c|}{$\mathrm{DN}(\mathrm{n}=49)$} & \multicolumn{2}{|c|}{ Proteinuric controls (MN/MCD, $\mathrm{n}=29$ ) } & $\mathrm{P}$ \\
\hline Age, years & $45.9 \pm 11.1$ & $53.9 \pm 9.8$ & $58.6 \pm 9.4^{*}$ & $46.9 \pm 14.1$ & 0.013 \\
\hline Male, n (\%) & $33(78.6)$ & $5(71.4)$ & $6(60.0)$ & $14(73.7)$ & 0.680 \\
\hline BMI, $\mathrm{kg} / \mathrm{m}^{2}$ & $25.2 \pm 4.4$ & $25.7 \pm 0.7$ & $24.9 \pm 2.2$ & $24.3 \pm 2.8$ & 0.754 \\
\hline $\mathrm{HbA1c}, \%$ & $7.8 \pm 1.9$ & $6.8 \pm 1.2$ & $7.6 \pm 1.5$ & - & 0.568 \\
\hline Diabetic retinopathy, n/n (\%) & $30 / 40(75.0)$ & $2 / 5(40.0)$ & $3 / 10(30.0)^{\star}$ & - & 0.016 \\
\hline Hypertension, n (\%) & $35(83.3)$ & $4(57.1)$ & $7(70.0)$ & $7(36.8)^{\star}$ & 0.004 \\
\hline Systolic BP, mmHg & $144.0 \pm 21.4$ & $125.9 \pm 10.3$ & $125.4 \pm 12.0^{\star}$ & $127.8 \pm 15.0^{\star}$ & 0.001 \\
\hline Urine protein, g/24 h & $5.7 \pm 3.7$ & $8.8 \pm 4.5$ & $4.7 \pm 3.4$ & $6.6 \pm 5.1$ & 0.203 \\
\hline Hematuria, n (\%) & 23(54.8) & $5(71.4)$ & $2(20.0)$ & $9(47.4)$ & 0.150 \\
\hline Urinary NAG, U/L & $39.5(21.3-55.8)$ & $37.0(20.0-61.8)$ & $37.0(22.5-66.0)$ & $59.0(33.5-124.5)$ & 0.697 \\
\hline Urinary $\alpha 1-M G, m g / L$ & $47.3(17.8-115.8)$ & $45.5(21.2-69.4)$ & $21.1(8.2-43.9)$ & 25.0 (17.9-79.9) & 0.248 \\
\hline
\end{tabular}

Data are presented as $\mathrm{n}(\%)$, or mean \pm SD or median (interquartile range). P values were estimated using ANOVA followed by Student's $t$ test, or Mann-Whitney rank sum test or chi-square test wherever applicable with Bonferroni correction. $\mathrm{P}$ values listed in the table are ANOVA comparing four groups and $\mathrm{P}<0.05$ are shown in bold. $\mathrm{P}>0.05$ indicates that there is no statistical significance among four groups. *, $\mathrm{P}<0.05$ compared to DN. BMI, body mass index; BP, blood pressure; DN, diabetic nephropathy; DM, diabetic mellitus; MN, membranous nephropathy; MCD, minimal change disease. Normal range: HbA1c, Glycated hemoglobin: 4.0-6.0\%; serum albumin: 40-55 g/L; serum creatinine: 44-133 $\mu \mathrm{mol} / \mathrm{L}$; urine protein: 0-0.15 g/24 h; urinary NAG, urinary N-acetyl-b-D-glucosaminidase: 0-21 U/L; urinary $\alpha 1-M G$, urinary $\alpha 1$-microglobulin: 0-12 mg/L.

patients with diabetes and those without (Table S1). We then explored the ability of urinary kidney MVs to discriminate between these two groups.

DN patients had higher urinary levels of MVs from podocytes, proximal tubular and endothelial cells compared with proteinuric controls (Table 2). Multivariate logistic analysis showed that podocyte nephrin ${ }^{+}$MVs could independently discriminate $\mathrm{DN}$ patients from proteinuric controls with an AUC of 0.815 (95\% CI: 0.713-0.911, $\mathrm{P}<0.001$, Table 3, Figure 3C). Furthermore, combining nephrin ${ }^{+} \mathrm{MVs}$ and diabetic retinopathy increased the AUC to 0.899 (95\% CI: $0.821-0.977, \mathrm{P}<0.001$ ) with $89.7 \%$ specificity and $88.9 \%$ sensitivity (Figure 3 C, Table S2).

\section{Association of urinary kidney MVs with pathological features in DN}

Forty-nine DN patients were pathologically grouped into
Class-I ( $\mathrm{n}=4)$, Class-II ( $\mathrm{n}=12)$, Class-III ( $\mathrm{n}=29)$ and ClassIV $(n=4)$, which mainly reflect the severity of DN featured glomerular lesions (Table S3). There was no significant difference in the amounts of urinary kidney MVs among the DN classes (not shown). The comprehensive urinary MV feature could hardly distinguish the DN patients with different pathological classifications (Figure 3D). Urinary podocyte MVs were not correlated with any of the glomerular lesions evaluated in DN patients (Figure $3 E$, Table S4).

Tubulointerstitial and vascular injury were prevalent in our DN patients. Their tubular injury score demonstrated positive relationships with urinary levels of tubular and endothelial MVs (Figure 3E, Table S4). Multivariant analysis showed that endothelial CD3 $1^{+} \mathrm{MVs}$ (OR 3.9, 95\% CI: 1.3-11.3, $\mathrm{P}=0.013$ ) could independently reflect the severity of tubular injury. Plus, the vascular score of DN patients positively correlated with tubular and endothelial 
A

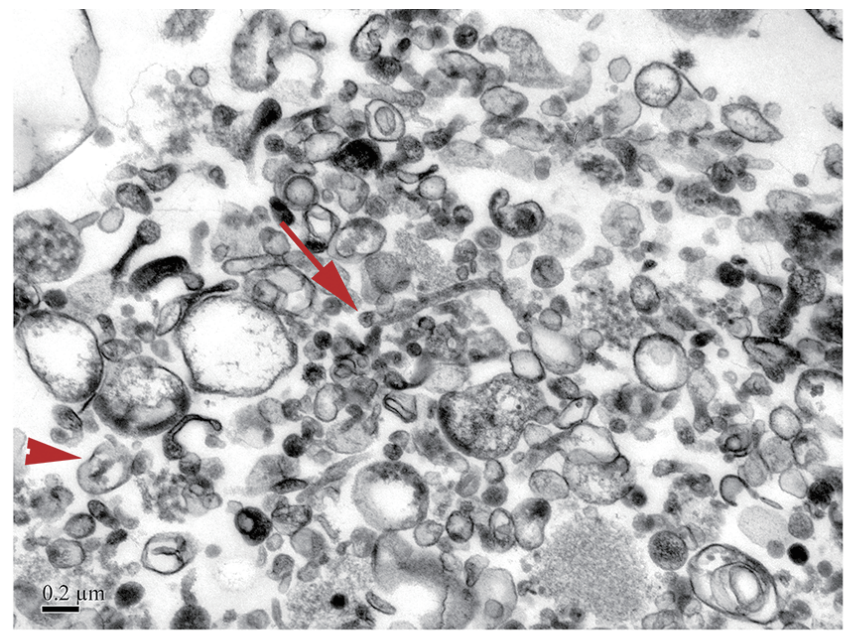

B

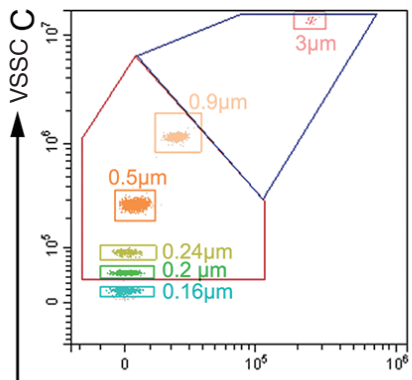

D
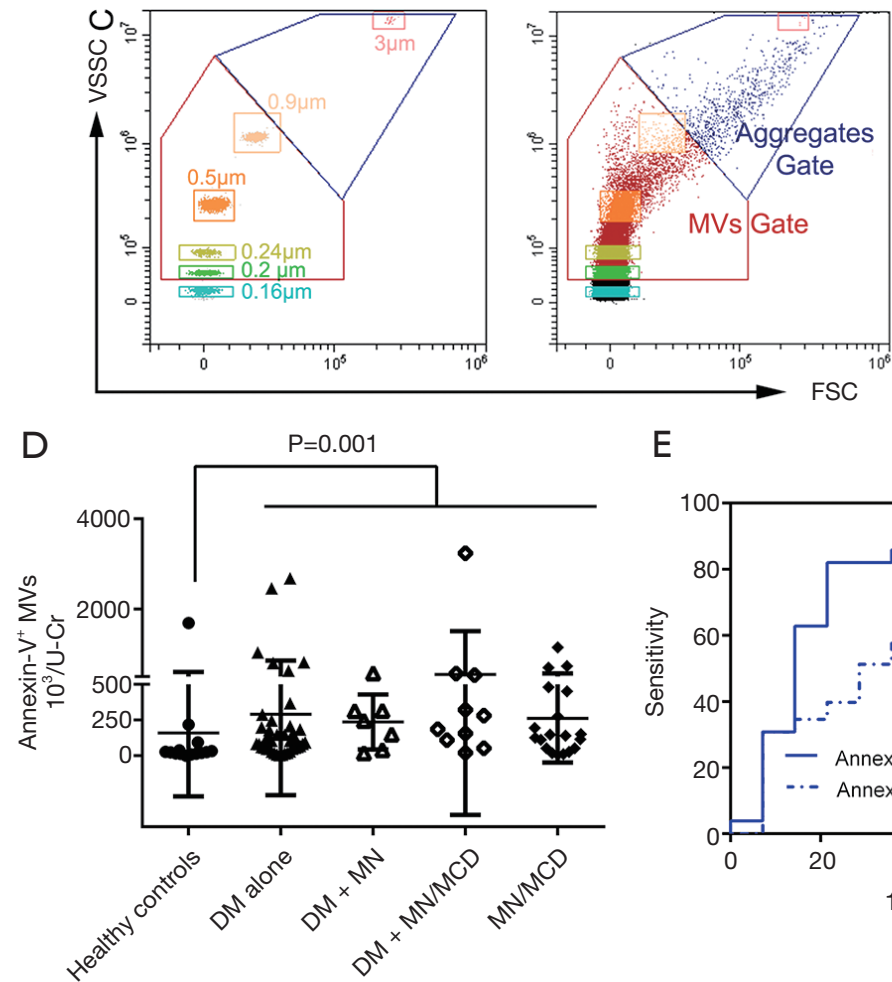

E

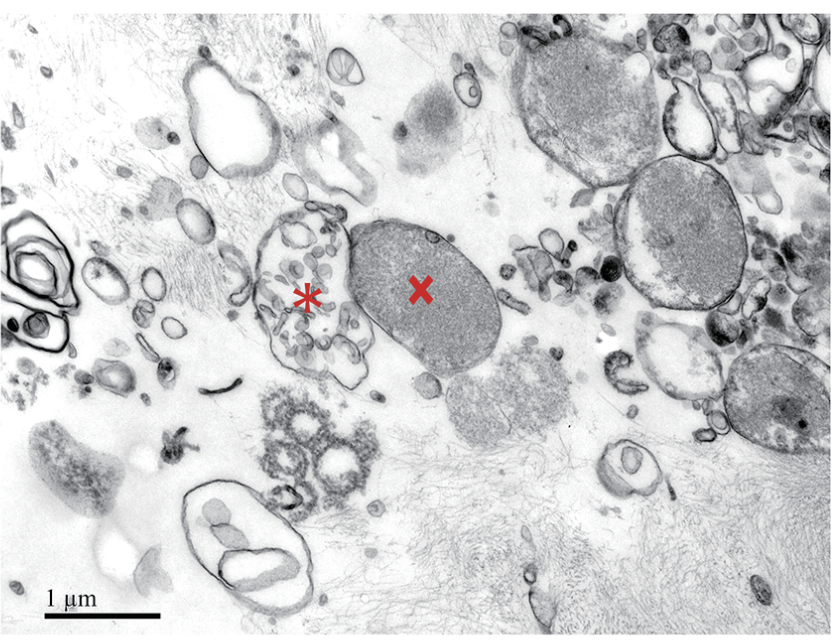

C

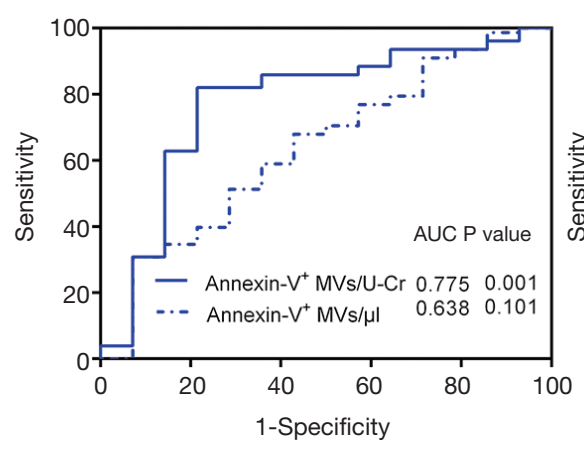

$\mathrm{F}$
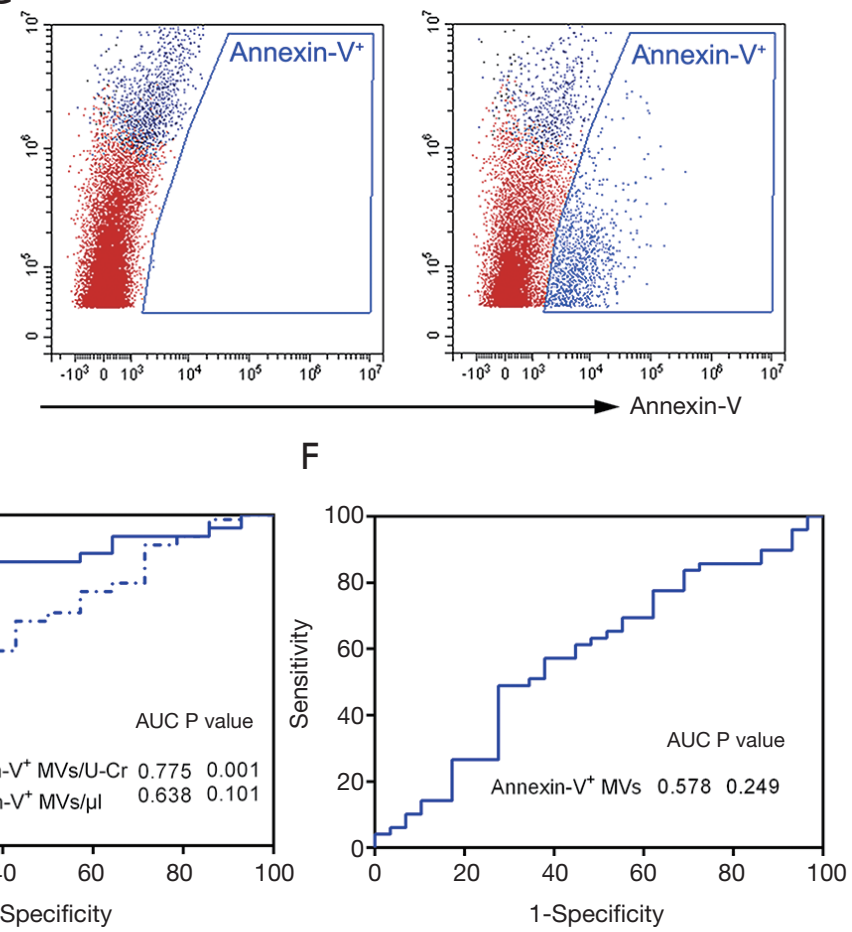

Figure 2 Detection of urinary MVs. (A) Transmission electron micrographs of heterogenous extracellular vesicles fractions from the urine of a DN patient. The arrow indicates an exosome, the arrowhead indicates a MV, the $\mathrm{X}$ symbol indicates an apoptotic body and the asterisk indicates a migrasome. (B) Gates for MVs were determined by Megamix beads (left) first and applied in urine samples (right) on FSC/VSSC channel. (C) On FITC/VSSC channel, annexin- $\mathrm{V}^{+}$MVs were identified, which is seen as a rightward shift. (D) The number of urinary annexin- $\mathrm{V}^{+} \mathrm{MVs}$ in healthy subjects and patients. (E-F) ROC curves of annexin- $\mathrm{V}^{+} \mathrm{MVs}$ to discriminate patients from healthy controls (E), and DN from patients (F). The corresponding AUCs for indicated biomarkers are also displayed in each panel. MVs, microvesicles; DN, diabetic nephropathy; DM, diabetic mellitus; MN, membranous nephropathy; MCD, minimal change disease; FSC, forward scatter; VSSC, violet side scatter; $\mathrm{U}-\mathrm{Cr}$, urinary creatinine; ROC, receiver operating characteristic; $\mathrm{AUC}$, area under the curve. 

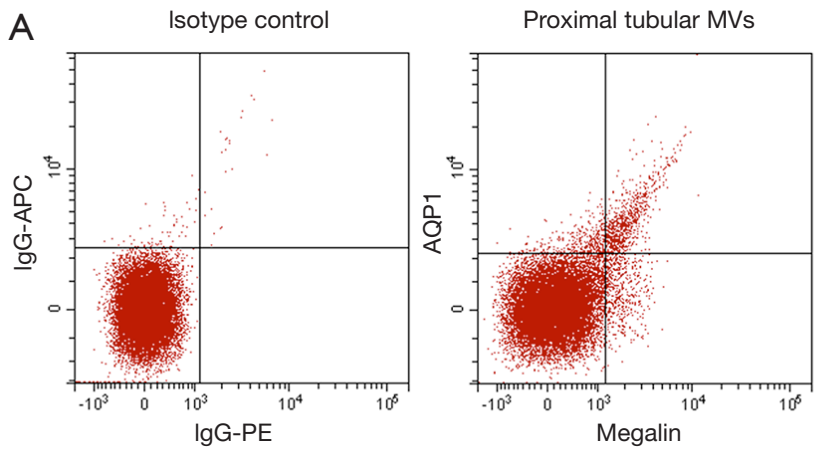

B

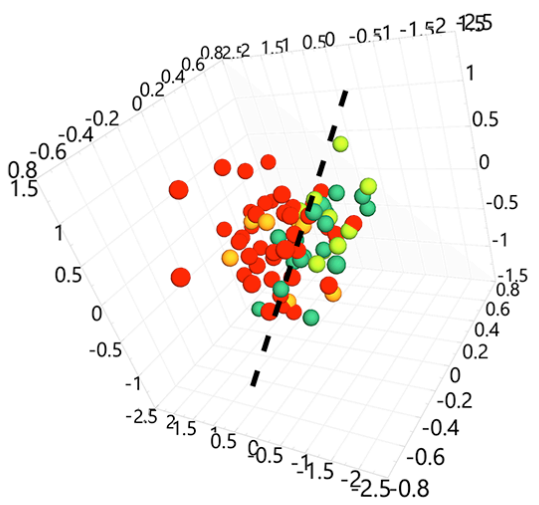

D

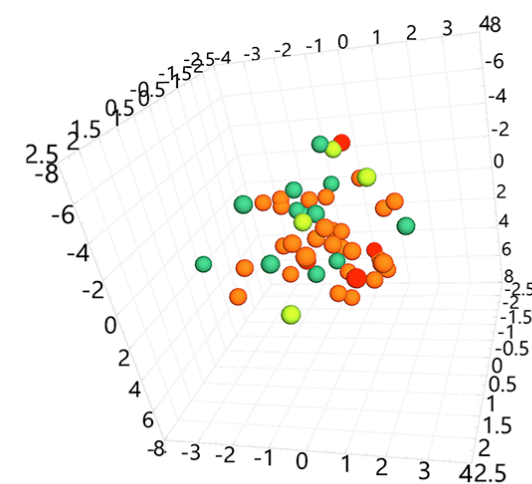

DM alone

$\mathrm{DM}+\mathrm{MN}$

$D M+M N / M C D$

$M N / M C D$
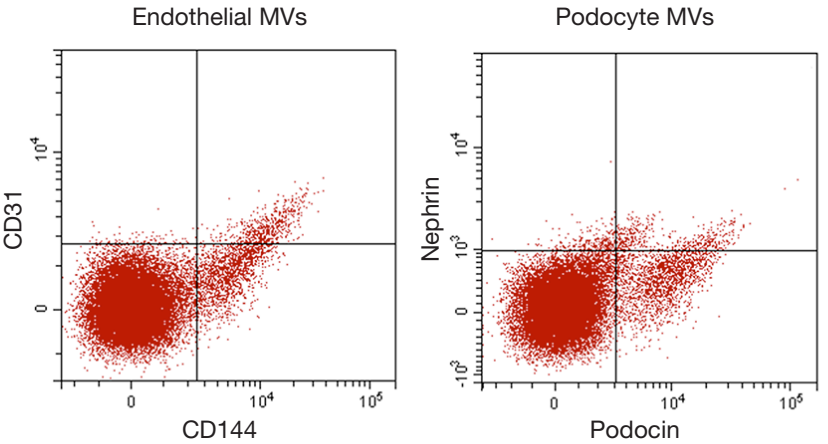

C
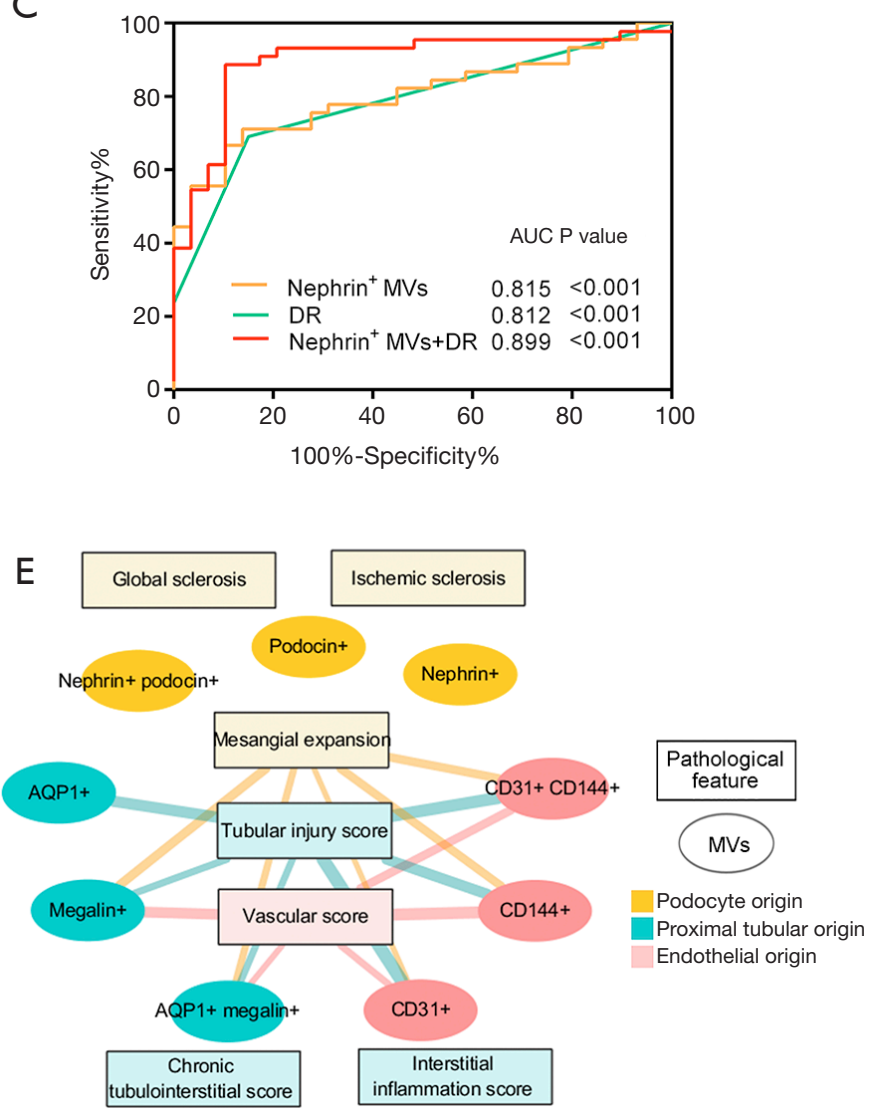

Figure 3 Diagnostic value of urinary kidney MVs and their association with pathological features. (A) Representative traces from flow cytometry analysis of podocyte, proximal tubular and endothelial MVs. The isotype control was shown as APC-conjugated IgG versus PEconjugated IgG. (B) PCA analysis showed that urinary MVs separated DN and proteinuric controls. (C) ROC curves of nephrin ${ }^{+}$MVs, DR and their combination to discriminate patients from healthy controls. (D) PCA analysis showed that urinary MVs could hardly discriminate DN patients with different pathological classifications. (E) Correlations between the urinary MV populations and pathological features. Edge width reflects the value of Spearman's correlation (range: 0.306-0.442, $\mathrm{P}<0.05$ ). MVs, microvesicles; DN, diabetic nephropathy; $\mathrm{AQP1}$, aquaporin 1; DM, diabetic mellitus; $\mathrm{MN}$, membranous nephropathy; $\mathrm{MCD}$, minimal change disease; ROC, Receiver-operating characteristic; AUC, area under the curve. PCA, principle component analysis. 
Table 2 Comparisons of urinary microvesicles between diabetic nephropathy and proteinuric controls

\begin{tabular}{|c|c|c|c|}
\hline & $\mathrm{DN}(\mathrm{n}=49)$ & Proteinuric controls $(n=29)$ & $P$ \\
\hline Nephrin $^{+}$ & $134.6(35.7-778.8)$ & $31.0(13.2-62.7)$ & $<0.001$ \\
\hline Podocin $^{+}$ & 221.4 (70.7-786.9) & $73.2(24.8-155.6)$ & $<0.001$ \\
\hline Nephrin $^{+}$podocin ${ }^{+}$ & $41.8(8.7-328.3)$ & $11.3(3.0-20.8)$ & $<0.001$ \\
\hline $\mathrm{AQP}^{+}$ & $234.1(111.1-962.6)$ & $82.9(41.1-240.7)$ & 0.005 \\
\hline Megalin $^{+}$ & $192.6(78.0-654.1)$ & $74.1(28.0-204.1)$ & 0.004 \\
\hline $\mathrm{AQP}^{+}$megalin $^{+}$ & $41.4(5.2-167.1)$ & $6.5(1.2-42.6)$ & 0.008 \\
\hline \multicolumn{4}{|l|}{ Endothelial MVs } \\
\hline $\mathrm{CD}_{3} 1^{+} \mathrm{CD} 144^{+}$ & $23.2(5.9-88.0)$ & $14.3(3.5-56.7)$ & 0.165 \\
\hline
\end{tabular}

MV levels are presented as median (interquartile range) $10 \%$ mol urinary creatinine. $\mathrm{P}$ values listed in the table are DN versus proteinuric controls and $\mathrm{P}<0.05$ are shown in bold. DN, diabetic nephropathy; AQP1, Aquaporin 1; MVs, microvesicles.

Table 3 Multivariant binary logistic analysis for urinary microvesicles predicting diabetic nephropathy

\begin{tabular}{|c|c|c|c|c|c|c|}
\hline & \multicolumn{3}{|c|}{ Univariant } & \multicolumn{3}{|c|}{ Multivariant } \\
\hline \multicolumn{7}{|l|}{ Podocyte MVs } \\
\hline $\lg \left(\right.$ nephrin $\left.^{+}\right)$ & 6.0 & $2.3-16.0$ & $<0.001$ & 134.5 & $2.7-6801.3$ & 0.014 \\
\hline $\lg \left(\right.$ podocin $\left.{ }^{+}\right)$ & 5.1 & $1.9-13.7$ & 0.001 & 3.0 & $0.1-103.4$ & 0.544 \\
\hline \multicolumn{7}{|l|}{ Proximal tubular MVs } \\
\hline $\lg \left(\mathrm{AQP} 1^{+}\right)$ & 3.0 & $1.3-6.8$ & 0.009 & 0.5 & $0.1-4.4$ & 0.547 \\
\hline $\lg \left(\right.$ megalin $\left.^{+}\right)$ & 2.9 & $1.3-6.3$ & 0.007 & 1.0 & $0.2-5.5$ & 0.979 \\
\hline $\lg \left(\mathrm{AQP} 1^{+}\right.$megalin $\left.{ }^{+}\right)$ & 2.2 & $1.2-4.0$ & 0.011 & 1.5 & $0.3-6.9$ & 0.620 \\
\hline $\lg \left(\mathrm{CD} 144^{+}\right)$ & 2.4 & $1.2-4.7$ & 0.016 & 1.2 & $0.3-4.5$ & 0.808 \\
\hline $\lg \left(\mathrm{CD} 31^{+} \mathrm{CD} 144^{+}\right)$ & 1.7 & $0.8-3.6$ & 0.167 & & & \\
\hline
\end{tabular}

$P$ values $<0.05$ are shown in bold. DN, diabetic nephropathy; AQP1, aquaporin 1; MVs, microvesicles; lg, log ${ }_{10}$; $\mathrm{Cl}$, confidence interval; OR, odds ratio. 
MVs, where the level of endothelial CD144 $4^{+}$MVs (OR 3.1, $95 \%$ CI: $1.2-8.4, \mathrm{P}=0.025)$ was the independent risk factor for vascular injury (Table S5). There was no association between urinary MVs and the degree of interstitial inflammation or chronic tubulointerstitial injury in DN (Figure 3E, Table S4).

\section{Significance of vascular injury in urinary tubular $M V$ release in $D N$}

As urine protein, interstitial inflammation and hypoxia induced by vascular injury are well-known insults contributing to tubular injury, we checked their effects on tubular MV release in our patients. In proteinuric controls, all the tubular MV populations were associated with the level of urinary protein excretion, indicating a potential causal effect of proteinuria on tubular MV production. To a lesser extent, urinary levels of the tubular MV populations correlated with the interstitial inflammation score, followed by the vascular score (Table 4, Table S6).

By contrast, in DN, urinary levels of the tubular MV populations were not associated with proteinuria or interstitial inflammation but rather positively associated with the vascular score (Table S6, Figure 3E). Significantly elevated tubular MVs were already detected in the DN patients with mild to moderate proteinuria $(<8.0 \mathrm{~g} / 24 \mathrm{~h})$ or interstitial inflammation (score $=1$ ) compared with the matched proteinuric controls (Table 4). Notably, these DN patients exhibited severer vascular injury than the proteinuric controls (Table S7). And after matching the vascular score of 1 or 2, the differences in urinary tubular MV levels diminished between DN patients and proteinuric controls.

\section{Discussion}

Approximately one-fifth of patients with diabetes develop $\mathrm{DN}$, and a significant proportion of these patients eventually progress to ESRD (21,22). With the high prevalence of diabetes and increasing complexity of kidney abnormalities in DN, it has become increasingly difficult to identify DN clinically (23). As DN, unlike other glomerulonephropathy, profoundly involves all components of the renal cortex (24), we explored the pathological relevance of urinary kidney-specific cell-derived MVs and found that podocyte MVs together with diabetic retinopathy could effectively discriminate $\mathrm{DN}$ from other types of proteinuric glomerulonephropathy. The model might be useful for physicians to make clinical decisions, for example, performing a kidney biopsy in a diabetic proteinuric patient to further confirm glomerulonephropathy or even considering immunosuppressive treatment when kidney biopsy is not applicable.

Previous studies showed that urinary podocyte MVs increased in advance of microalbuminuria, suggesting the use of podocyte MVs for identifying diabetic individuals at risk of developing podocytopathy $(14,25)$. However, in our DN patients, urinary podocyte MVs were not relevant to any glomerular features. As these patients already exhibited apparent proteinuria and wellestablished DN histological abnormalities, we assume that podocyte MV release had been increased to high levels at the early stage of DN and remained afterwards. Therefore, urinary podocyte MVs could serve as a sensitive marker for detection of DN but might not be able to assess glomerular damage in advanced DN.

DN patients have advanced tubular injury which could occur very early and associates with renal survival in DN $(26,27)$. The related pathogenesis may be, at least partly, independent of glomerular damage $(6,28,29)$. In the current study, using urinary proximal tubular MVs as the indicators for tubular injury, we attempted to figure out the main culprit for tubular injury in DN. In proteinuric controls, tubular MV levels correlated primarily with the level of proteinuria, followed by the degree of interstitial inflammation and vascular injury. This support the fact that proteinuria is nephrotoxic to tubules $(30,31)$, while interstitial inflammation and vascular ischemic insults could be synergistic factors. By contrast, in DN, tubular MVs associated with neither proteinuria nor interstitial inflammation. In fact, tubular MV levels were already significantly higher in DN patients than in proteinuric controls when proteinuria was at an asymptomatic level $(<4 \mathrm{~g} / 24 \mathrm{~h})(31)$ or interstitial inflammation was low-grade $(<25 \%)$, indicating a culprit other than these two. Notably, tubular MV levels correlated with the severity of renal vascular injury. After matching the degree of vascular injury, differences in tubular MV levels between DN patients and proteinuric controls diminished. These data highlight the importance of insults secondary to vascular injury contributing to tubular injury in DN.

Extensive endothelial injury is a distinct feature of DN (5), which involves both glomerular capillaries and vessels in the kidney (32-34). The vascular score in our study refers to arteriolar hyalinosis and arteriosclerosis, representing a constant local ischemic and hypoxic state. 


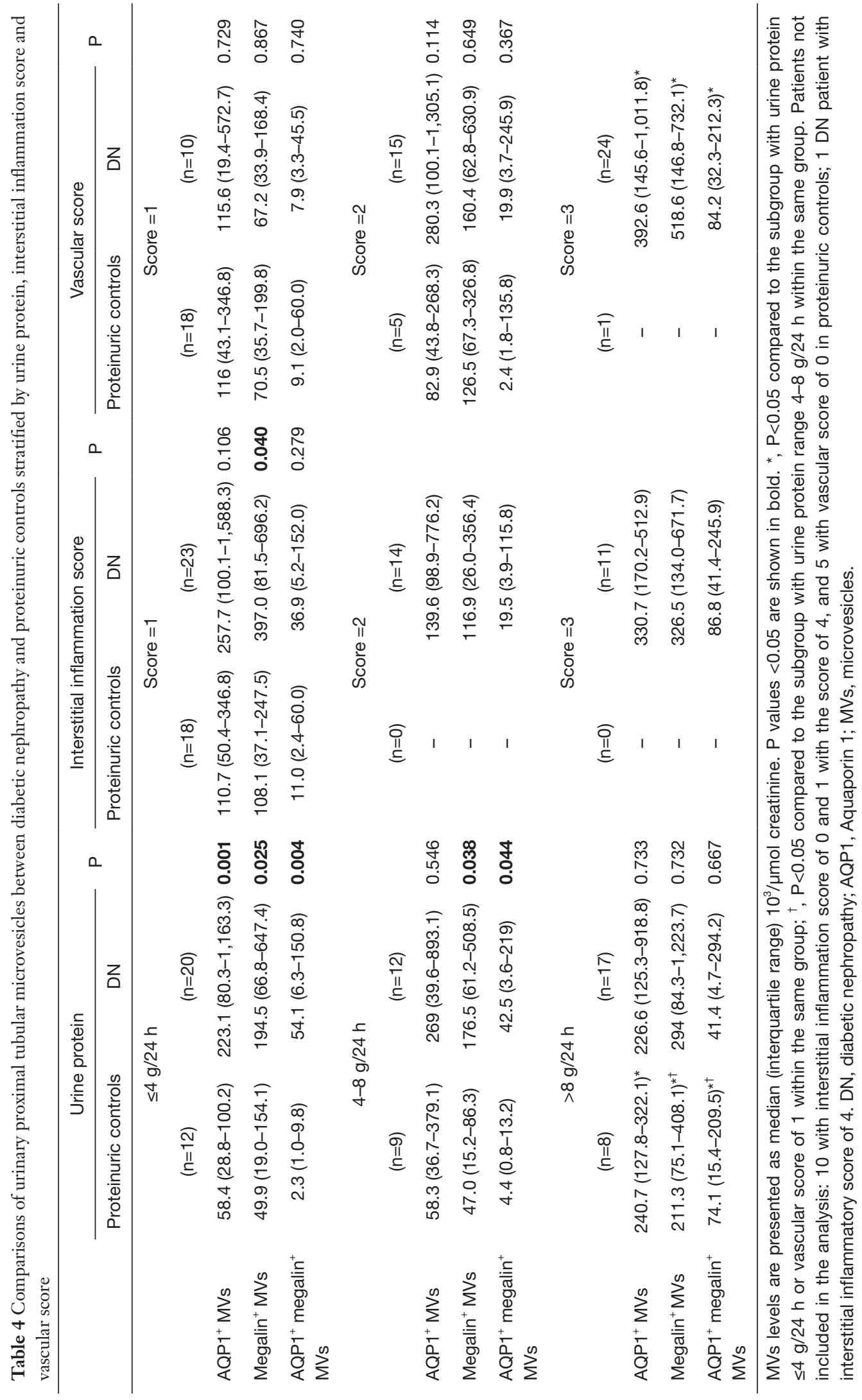


Vascular injury was markedly severer in $\mathrm{DN}$ patients than in proteinuric controls, even when urine protein and interstitial inflammation were low-grade, suggesting that vascular injury occurs early and progresses profoundly in DN patients. During the past decade, substantial researches explored the mechanistic role of endothelial impairment in the progression of $\mathrm{DN}(5,35)$. Recently, striking improvement has been achieved in treating patients with a selective endothelin A receptor, which provides an effective therapeutic choice in DN patients at risk of ESRD (36). Our study found that urinary levels of endothelial MVs not only independently reflected vascular injury but also correlated with the degree of glomerular mesangial expansion and tubular injury. Thus, using urinary endothelial MVs as biomarkers for evaluating renal vascular involvement might help identify the subgroup of DN patients who would benefit from endothelial targeting therapies.

The main strength of the study is that it is based on a cohort of biopsy-proven DN and other glomerulonephropathy, which help accurate evaluation of the diagnostic ability and enabled direct comparisons of various MV populations with pathological features. Our study has limitations. First, although the sample size was sufficient for statistical analysis, it was relatively small and the diagnostic value of the urinary MVs should be carefully interpreted and needs to be reproduced in larger sample sizes and different ethnic groups, and the test should be validated by regulatory organizations for further development. Second, selection bias largely originated from diabetic patients prescribed kidney biopsy. DN patients in our study had proteinuria. As a fraction of diabetic patients had declined renal function before proteinuria (37), whether our results were applicable to these patients should be further tested. Third, age and blood pressure could be possible confounders as there were differences among various subgroups as shown in Table 1. But hypertension is also a presentation of vascular malfunction. Finally, due to the cross-sectional study design, we were not able to investigate whether urinary MVs could predict renal outcomes in DN.

In summary, our study demonstrated that urinary kidney MVs might serve as noninvasive biomarkers for DN. Their elevated levels reflected the severity of pathological lesions, and a model combining podocyte and diabetic retinopathy could help identify DN.

\section{Acknowledgments}

Funding: This study was supported by grants from the
National Key Research and Development Program of China (2018YFC1314004), the National Natural Science Foundation of China (No. 91742205 and No. 81625004), the National Science and Technology Major Projects for "Major New Drugs Innovation and Development" of China (No. 2017ZX09304028) and Peking University Clinical Scientist Program by the Fundamental Research Funds for the Central Universities.

\section{Footnote}

Reporting Checklist: The authors have completed the STARD reporting checklist. Available at http://dx.doi.org/10.21037/ atm-20-441

Data Sharing Statement: Available at http://dx.doi. org/10.21037/atm-20-441

Peer Review File: Available at http://dx.doi.org/10.21037/ atm-20-441

Conflicts of Interest: All authors have completed the ICMJE uniform disclosure form (available at http://dx.doi. org/10.21037/atm-20-441). The authors have no conflicts of interest to declare.

Ethical Statement: The authors are accountable for all aspects of the work in ensuring that questions related to the accuracy or integrity of any part of the work are appropriately investigated and resolved. The research was in compliance of the Declaration of Helsinki (as revised in 2013), and approved by the Committee on Research Ethics of Peking University First Hospital (NO. 20171280). All participants gave written informed consent.

Open Access Statement: This is an Open Access article distributed in accordance with the Creative Commons Attribution-NonCommercial-NoDerivs 4.0 International License (CC BY-NC-ND 4.0), which permits the noncommercial replication and distribution of the article with the strict proviso that no changes or edits are made and the original work is properly cited (including links to both the formal publication through the relevant DOI and the license). See: https://creativecommons.org/licenses/by-nc-nd/4.0/.

\section{References}

1. Guilbert JJ. The World Health Report 2006: working 
together for health. Educ Health (Abingdon) 2006;19:385-7.

2. Zhang L, Long J, Jiang W, et al. Trends in Chronic Kidney Disease in China. N Engl J Med 2016;375:905-6.

3. Tervaert TW, Mooyaart AL, Amann K, et al. Pathologic classification of diabetic nephropathy. J Am Soc Nephrol 2010;21:556-63.

4. Dalla Vestra M, Masiero A, Roiter AM, et al. Is podocyte injury relevant in diabetic nephropathy? Studies in patients with type 2 diabetes. Diabetes 2003;52:1031-5.

5. Nakagawa T, Tanabe K, Croker BP, et al. Endothelial dysfunction as a potential contributor in diabetic nephropathy. Nat Rev Nephrol 2011;7:36-44.

6. Tang SC, Yiu WH, Lin M, et al. Diabetic nephropathy and proximal tubular damage. J Ren Nutr 2015;25:230-3.

7. Das U, Dakshinamurty KV, Prayaga A, et al. Nondiabetic kidney disease in type 2 diabetic patients: A single center experience. Indian J Nephrol 2012;22:358-62.

8. Fan JZ, Wang R. Non-diabetic renal disease in patients with type 2 diabetes: a single centre study. Intern Med J 2018;48:451-6.

9. Erdogmus S, Kiremitci S, Celebi ZK, et al. Non-Diabetic Kidney Disease in Type 2 Diabetic Patients: Prevalence, Clinical Predictors and Outcomes. Kidney Blood Press Res 2017;42:886-93.

10. Horvatic I, Tisljar M, Kacinari P, et al. Non-diabetic renal disease in Croatian patients with type 2 diabetes mellitus. Diabetes Res Clin Pract 2014;104:443-50.

11. Wang J, Han Q, Zhao L, et al. Identification of clinical predictors of diabetic nephropathy and non-diabetic renal disease in Chinese patients with type 2 diabetes, with reference to disease course and outcome. Acta Diabetol 2019;56:939-46.

12. van Niel G, D'Angelo G, Raposo G. Shedding light on the cell biology of extracellular vesicles. Nat Rev Mol Cell Biol 2018;19:213-28.

13. Karpman D, Stahl AL, Arvidsson I. Extracellular vesicles in renal disease. Nat Rev Nephrol 2017;13:545-62.

14. Lytvyn Y, Xiao F, Kennedy CR, et al. Assessment of urinary microparticles in normotensive patients with type 1 diabetes. Diabetologia 2017;60:581-4.

15. Kwon SH, Woollard JR, Saad A, et al. Elevated urinary podocyte-derived extracellular microvesicles in renovascular hypertensive patients. Nephrol Dial Transplant 2017;32:800-7.

16. Gilani SI, Anderson UD, Jayachandran M, et al. Urinary Extracellular Vesicles of Podocyte Origin and Renal Injury in Preeclampsia. J Am Soc Nephrol 2017;28:3363-72.
17. Sun IO, Santelli A, Abumoawad A, et al. Loss of Renal Peritubular Capillaries in Hypertensive Patients Is Detectable by Urinary Endothelial Microparticle Levels. Hypertension 2018;72:1180-8.

18. Jayachandran M, Lugo G, Heiling H, et al. Extracellular vesicles in urine of women with but not without kidney stones manifest patterns similar to men: a case control study. Biol Sex Differ 2015;6:2.

19. Turco AE, Lam W, Rule AD, et al. Specific renal parenchymal-derived urinary extracellular vesicles identify age-associated structural changes in living donor kidneys. J Extracell Vesicles 2016;5:29642.

20. Wisgrill L, Lamm C, Hartmann J, et al. Peripheral blood microvesicles secretion is influenced by storage time, temperature, and anticoagulants. Cytometry A 2016;89:663-72.

21. King P, Peacock I, Donnelly R. The UK prospective diabetes study (UKPDS): clinical and therapeutic implications for type 2 diabetes. Br J Clin Pharmacol 1999;48:643-8.

22. Dean J. Organising care for people with diabetes and renal disease. J Ren Care 2012;38 Suppl 1:23-9.

23. Baudy A, Batuman V. Non-diabetic renal disease in diabetic patients: How to identify? When to biopsy? J Diabetes Complications 2015;29:613-4.

24. Fioretto P, Mauer M. Diabetic nephropathy: diabetic nephropathy-challenges in pathologic classification. Nat Rev Nephrol 2010;6:508-10.

25. Burger D, Thibodeau JF, Holterman CE, et al. Urinary podocyte microparticles identify prealbuminuric diabetic glomerular injury. J Am Soc Nephrol 2014;25:1401-7.

26. An Y, Xu F, Le W, et al. Renal histologic changes and the outcome in patients with diabetic nephropathy. Nephrol Dial Transplant 2015;30:257-66.

27. Taft JL, Nolan CJ, Yeung SP, et al. Clinical and histological correlations of decline in renal function in diabetic patients with proteinuria. Diabetes 1994;43:1046-51.

28. Maezawa Y, Takemoto M, Yokote K. Cell biology of diabetic nephropathy: Roles of endothelial cells, tubulointerstitial cells and podocytes. J Diabetes Investig 2015;6:3-15.

29. Gilbert RE, Cooper ME. The tubulointerstitium in progressive diabetic kidney disease: more than an aftermath of glomerular injury? Kidney Int 1999;56:1627-37.

30. Lv LL, Feng Y, Wen Y, et al. Exosomal CCL2 from Tubular Epithelial Cells Is Critical for Albumin-Induced Tubulointerstitial Inflammation. J Am Soc Nephrol 2018;29:919-35. 
31. Cattran DC. Idiopathic membranous glomerulonephritis. Kidney Int 2001;59:1983-94.

32. Jennette JC, Silva FG, Olson JL, et al. Heptinstall's Pathology of the Kidney. 7th edition. Waltham, MA: Wolters Kluwer Health, 2014.

33. Shi Y, Vanhoutte PM. Macro- and microvascular endothelial dysfunction in diabetes. J Diabetes 2017;9:434-49.

34. Goligorsky MS. Vascular endothelium in diabetes. Am J Physiol Renal Physiol 2017;312:F266-75.

35. Blantz RC. Phenotypic characteristics of diabetic kidney

Cite this article as: Cai FH, Wu WY, Zhou XJ, Yu XJ, Lv JC, Wang SX, Liu G, Yang L. Diagnostic roles of urinary kidney microvesicles in diabetic nephropathy. Ann Transl Med 2020;8(21):1431. doi: 10.21037/atm-20-441 involvement. Kidney Int 2014;86:7-9.

36. Heerspink HJL, Parving HH, Andress DL, et al. Atrasentan and renal events in patients with type 2 diabetes and chronic kidney disease (SONAR): a doubleblind, randomised, placebo-controlled trial. Lancet 2019;393:1937-47.

37. Porrini E, Ruggenenti P, Mogensen CE, et al. Nonproteinuric pathways in loss of renal function in patients with type 2 diabetes. Lancet Diabetes Endocrinol 2015;3:382-91. 